Pacific Journal of Mathematics

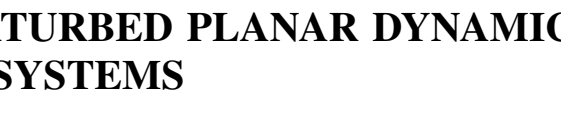




\section{TRANSVERSALLY PERTURBED PLANAR DYNAMICAL SYSTEMS}

\section{ROGer C. MCCANN}

This paper investigates the behavior of limit cycles of a planar dynamical system which has been perturbed transversally. In particular, it is shown that if $C$ is a limit cycle of the unperturbed dynamical system, then there are limit cycles of the perturbed dynamical systems arbitrarily close to $C$. Also, if $C$ is an exterior limit cycle of the unperturbed dynamical system, then there is an outer neighborhood of $C$ which consists solely of cycles of the perturbed dynamical systems.

In what follows $R$ and $R^{2}$ will denote the reals and the plane respectively.

A dynamical system is an ordered pair $(X, \pi)$ consisting of a topological space $X$ and a mapping $\pi$ of $X \times R$ into $X$ such that (where $x \pi t=\pi(x, t)$ )
(i) $\quad x \pi t=x$ for all $x \in X$
(ii) $(x \pi t) \pi s=x \pi(t+s)=x_{\pi}(s+t)$ for all $x \in X$ and $s, t \in R$
(iii) $\pi$ is continuous in the product ropology.

A point $x \in X$ is called critical if and only if $x \pi t=x$ for every $t \in R$. A point $x \in X$ is called periodic if and only if $x$ is noncritical and $x \pi t=x$ for some $t>0$; if $X$ is Hausdorff the least such $t$ is called the fundemental pariod of $x$. If $x$ is periodic, $x \pi R$ is called a cycle. A cycle is a simple closed curve. Hence, if $C$ is a cycle of a planar dynamical system $\left(R^{2}, \pi\right)$, then $C$ decomposes $R^{2}$ into two components; one bounded and denoted by int $C$; the other unbounded and denoted by ext $C$. A subset $A$ of $X$ is called a trajectorial arc if and only if there is an $x \in X$ and a compact interval $[a, b], a \neq b$, such that $A=x \pi[a, b]$.

Let $\left(R^{2}, \pi\right)$ be a dynamical system. A subset $T$ of $R^{2}$ is called a transversal if and only if

(i) $T$ is homeomorphic with either $[0,1]$ or $S^{1}$, the 1-sphere

(ii) there is an $\varepsilon>0$ such that $T \cap(T \pi t)=\varnothing$ for $0<|t| \leqq \varepsilon$.

Our investigation depends heavily upon the following three propositions which may be found in [2, VII, 4.4], [2, VII, 4.7], and [2, VII, 4.8] respectively.

Proposition A. Let $C$ be a trajectory and $T$ a transversal of a planar dynamical system. If $C$ or $T$ is a closed curve, they have at most one intersection point; if both are closed curves, they do not 
intersect.

Proposition B. Let $C \cup T$ be a simple closed curve with $C$ a trajectorial are and $T$ a transversal of a planar dynamical system. Then one component of $R^{2}-(C \cup T)$ is positively invariant, the second is negatively invariant, and neither is invariant. The result is also valid if $C=\varnothing$.

Proposition C. In a planar dynamical system the interior of each cycle, closed transversal, or simple closed curve consisting of a transversal and a trajectorial arc, all contain a critical point.

We are interested in studying a family of dynamical systems which is defined as follows. Let $\pi: R^{2} \times R \times R \rightarrow R$ be a mapping continuous in the product topology such that

(i) for each $a \in R$ the mapping $\pi_{a}: R^{2} \times R \rightarrow R^{2}$ defined by $\pi_{a}(x, t)=\pi(x, t, a)$ defines a dynamical system on $R^{2}$.

(ii) critical points of the dynamical systems are independent of the index.

(iii) the noncritical trajectories of $\pi_{a}$ are transversal to the noncritical trajectories of $\pi_{b}$ if $a \neq b$, i.e., if $T$ is a trajectorial arc of $\pi_{a}$, then $T$ is a transversal with respact to $\pi_{b}$ if $a \neq b$.

$C_{a}(x), C_{a}^{+}(x), L_{a}^{+}(x)$, and $L_{a}^{-}(x)$ will denote the trajectory, positive semitraiectory, positive limit set, and negative limit set, respectively, of $x$ with respect to $\pi_{a}$. The family of all trajectories of $\pi_{a}$, a fixed, will be called a system and the family of all trajectories will be called a complete family.

In [1] and [4] sufficient conditions are given which assure that the differential equations

$$
\dot{x}=P(x, y, a), \quad \dot{y}=Q(x, y, a),
$$

where the dots stand for differentiation with respect to the independent vaiable $t$ and $a$ is a paremeter, define a complete family.

Immediate consequences of Propositions $\mathrm{A}$ and $\mathrm{C}$ are the following two propositions.

Proposition 1. Cycles of distinct systems of a complete family do not interset.

Proposition 2. Let $x$ be a noncritical point of a complete family, $a \neq b$, and suppose that $C_{a}(x)$ and $C_{b}(x)$ have a point $y, y \neq x$, in common. If the trajectorial arcs of $C_{a}(x)$ and $C_{b}(x)$ connecting the points $x$ and $y$ have only their endpoints in common, then the region 
bounded by these trajectorial arcs contains a critical point.

Proposition 3. Let $C$ be a cycle of $\pi_{a}$. Then int $C$ is positively invariant with respect to $\pi_{b}$ for all $b>a$ or int $C$ is negatively invariant with respect to $\pi_{b}$ for all $b>a$, but in neither case is int $C$ invariant with respect to $\pi_{b}$ for any $b>a$. A similar result holds for $b<a$.

Proof. Consider the sets

$A=\left\{b \in(a,+\infty):\right.$ int $C$ is positively invariant with respect to $\left.\pi_{b}\right\}$

$B=\left\{b \in(a,+\infty): \operatorname{int} C\right.$ is negatively invariant with respect to $\left.\pi_{b}\right\}$. By Proposition $\mathrm{B}$, int $C$ is positively invariant or negatively invariant, but not both, with respect to each $\pi_{b}, b>a$. Thus $A \cup B=(a,+\infty)$ and $A \cap B=\varnothing$. We now show that both $A$ and $B$ are open. If $c \in(\alpha,+\infty)-A=B$, then there exist $x \in \operatorname{int} C$ and $t>0$ such that $x \pi_{c} t \in \operatorname{ext} C$. Since $\pi$ is continuous $x \pi_{b} t \in \operatorname{ext} C$ for all $b$ sufficiently close to $c$. Hence $B$ is open. Similarly $A$ is open. The connectivity of $(a,+\infty)$ implies either $A$ or $B$ must be empty. This completes the proof.

Proposition 4. Let $C$ be a cycle of $\pi_{a}$. If int $C$ is positively invariant with respect to every $\pi_{b}, b>a$, then ext $C$ is positively invariant with respect to every $\pi_{b}, b<a . A$ similar result holds if $b>a$ and $b<a$ are interchanged.

Proof. Let $x \in C$ and $T$ be a trajectorial arc of $C_{c}(x), c>a$, which contains $x$ as a nonend point. Then $T$ is a transversal with respect to $\pi_{b}, b \neq c$, Moreover, if $\tau$ is the fundamental period of $C$, then $T \pi_{a}[-\tau, \tau]$ is a connected neighborhood of $C$ which contains no critical points. Choose a neighborhood $U$ of $x, 0<\sigma<|c-a|$, and $0<\varepsilon<\tau$ so small that $U \pi_{b}[-\varepsilon, \varepsilon] \subset T \pi_{a}[\tau, \tau]$ for all $b \in[a-\sigma, a+\sigma]$. This is possible because $\pi$ is continuous. We can now define a mapping $h$ of $[a, a+\sigma]$ into $S=\left\{x \pi_{b} \varepsilon: b \in[a, a+\sigma]\right\}$ by $h(b)=x \pi_{b} \varepsilon . \quad h$ is continuous since $\pi$ is continuous. For $b \neq d, x \pi_{b} \varepsilon$ and $x \pi_{d} \varepsilon$ cannot be equal; for if they were Proposition 2 would imply that $T \pi_{a}[-\tau, \tau]$ contains a critical point. Hence $h$ is one-to-one. Obviously, $h$ is an onto mapping. A one-to-one continuous mapping of a compact space onto a Hausdorff space is a homeomorphism. Thus $S$ is an arc. Since int $C$ is, by assumption, positively invariant with respect to $\pi_{b}, b>a$, we have $S \subset \overline{\operatorname{int} C}$. Moreover, $\left(x \pi_{a}[0, \varepsilon]\right) \cup S \cup\left(x \pi_{a+o}[0, \varepsilon]\right)$ forms a simple closed curve $J$ such that int $J \subset T \pi[-\tau, \tau]$ and $\overline{\operatorname{int} J}$ is a neighborhood of $x \pi_{a} \varepsilon / 2$ relative to $\overline{\operatorname{int} C}$. Let $y \in \operatorname{int} J$ and set 


$$
J_{t}=\left(x \pi_{a}[0, t]\right) \cup\left(x \pi_{a+\sigma}[0, t]\right) \cup\left\{x \pi_{b} t: b \in[a, a+\sigma]\right\} .
$$

For each $t, 0<t<\varepsilon, J_{t}$ is a simple closed curve. Since $\pi$ is continuous, $y \in \operatorname{ext} J_{t}$ for $t$ sufficiently small. But for $t=\varepsilon, y \in \operatorname{int} J_{\varepsilon}=$ int $J$. The continuity of $\pi$ implies there is an $s \in(0, \varepsilon)$ such that $y \in J_{s}$. By the construction of $J_{s}$ and since $y \in \operatorname{int} J, y$ must be an element of $\left\{x \pi_{b} s: b \in[a, a+\sigma]\right\}$. This shows that int $\bar{J}$ consists solely of trajectorial arcs from the systems $\pi_{b}, b \in[a, a+\sigma]$.

Now let $V$ be a neighborhood of $x \pi_{a} \varepsilon / 2$ such that $V \cap \operatorname{int} C \subset \operatorname{int} J$. Then there is an $\alpha, 0<\alpha<\sigma$, such that $x \pi_{b} \varepsilon / 2 \in V$ for all $b \in[\alpha-\alpha, 0]$. For $b \in[a-\alpha, 0), x \pi_{b} \varepsilon / 2$ cannot be an element of $\overline{\operatorname{int} C}$ for then

$$
x \pi_{b} \varepsilon / 2 \subset V \cap \operatorname{int} C \subset \operatorname{int} J \subset \bigcup\left\{x \pi_{c}[0, \varepsilon]: c \in[a, a+\sigma]\right\} .
$$

This, by Proposition 2, implies that $T \pi_{a}[-\tau, \tau]$ contains a critical point. Hence for $b \in[a-\alpha, 0)$ we have $x \pi_{b} \varepsilon / 2 \in \operatorname{ext} C$ and therefore, by Proposition $B, C_{b}^{+}(x) \subset \operatorname{ext} C$. Proposition 3 now implies the desired result.

Proposition 4 allows us to assume throughout the remainder of the paper that if $C$ is a given cycle of $\pi_{a}$, then int $C$ is positively invariant with respect to every $\pi_{b}, b<a$, and negatively invariant with respect to every $\pi_{b}, b>a$. If the opposite invariance properties hold, the following propositions remain valid after the obvious modifications are made.

Definition 5. Let $C$ be a cycle of $\pi_{a}$. If there is an $x \in \operatorname{ext} C$ such that $L_{a}^{+}(x)=C$ or $L_{a}^{-}(x)=C$, then $C$ is called an external limit cycle or a external negative limit cycle, respectively. Similarily, if there is an $x \in \operatorname{int} C$ such that $L_{a}^{+}(x)=C$ or $L_{a}^{-}(x)=C$, then $C$ is called an internal limit cycle or a internal negative limit cycle, respectively.

Definition 6. Let $U$ be a neighborhood of a simple closed curve $C$. Then $U$-int $C$ and $U$-ext $C$ are called an outer neighborhood and an inner neighborhood, respectively, of $C$.

Proposition 7. Let $C$ be an external limit cycle of $\pi_{a}$. Then, given any outer neighborhood $U$ of $C$, there exists an $\varepsilon>0$ such that, for each $b \in[a, a+\varepsilon], U$ contains both an external limit cycle and an internal limit cycle of $\pi_{b}$ (the two cycles may coincide). A similar result holds for $C$ an internal limit cycle and $b \in[a-\varepsilon, a]$.

Proof. Let $V \subset U$ be an outer neighborhood of $C$ containing no critical points and such that int $C \cup V$ is simply connected. Let $x \in C$, $y \in \operatorname{ext} C$ be such that $L_{a}^{+}(y)=C$, and $T \subset V$ be a trajectorial arc of 
$C_{c}(x), c<a$, containing $x$ as an endpoint. Then $T$ is a transversal with respect to $\pi_{b}, b \neq c$. Since $L_{a}^{+}(y)=C, y \in \operatorname{ext} C$, and $V$ is an outerneighborhood of $C$, there is a $\tau>0$ such that $y \pi_{a}[\tau,+\infty) \subset V$. Let $y_{1}, y_{2} \in y \pi_{a}[\tau,+\infty)$ be consecutive points of intersection between $C_{a}^{+}(\mathrm{y})$ and $T$ with $y_{2} \in C_{a}^{+}\left(y_{1}\right)$. Then the trajectial arc of $C_{a}^{+}(y)$ and the subarc of $T$ connecting $y_{1}$ and $y_{2}$ form a simple closed curve $J \subset V$ such that int $J$-int $C \subset V$. Now $L_{a}^{+}\left(y_{1}\right)=L_{a}^{+}(y)=C \subset \operatorname{int} J$ and Proposition B imply $y_{2} \pi_{a}(0,+\infty) \subset \operatorname{int} J$. Since $y_{2} \in C_{a}^{+}\left(y_{1}\right)$ and $\pi$ is continuous there is an $\varepsilon>0$ such that $C_{b}^{+}\left(y_{1}\right)$ intersects int $J$ for $|b-a|<\varepsilon$. If $y_{1} \pi_{b} t \in$ int $J$ for some $t>0$, then $y_{1} \pi_{b}[t, \infty)$ must be a subset of int $J$; for if it were not $y_{1} \pi_{b}[t, \infty)$ would intersect $J$ and Proposition 2 would imply int $J$-int $C$, and hence $V$, contains a critical point. Moreover, by the continuity of $\pi$, and the fact $L_{a}^{+}\left(y_{1}\right)=C$, we may assume that $\varepsilon$ was chosen so small that $C_{b}^{+}\left(y_{1}\right),|b-a|<\varepsilon$, intersects $T$ at least twice between $y_{2}$ and $x$. This is true because $C_{a}^{+}\left(y_{1}\right)$ intersects $T$ infinitely many times and the only limit point of the intersections is $x,[2$, VIII, 1.2] and [2, VIII, 1.5]. The trajectorial arc connecting two such consecutive points of intersection and the corresponding subarc of $T$ form a simple closed curve $J_{b}$ such that int $J_{b} \subset$ int $J$ and int $J_{b}$-int $C \subset V$. Moreover, int $J_{b}$ is positively invariant with respect to $\pi_{b}$ by Propoisition B. Thus int $J_{b}$ and ext $C$ are both positively invariant with respect to $\pi_{b}$. Hence int $J_{b}$-int $C$ is positively invariant, so that $\mathrm{C}_{b}^{+}(x) \subset \overline{\operatorname{int} J_{b}-\operatorname{int} C}$ which is compact and contains no critical points. By the Poincaré-Bendixson Theorem, [2, VII, 1.14], $L_{b}^{+}(x)$ is a cycle $C_{b}$. Since int $J_{b}$ is positively invariant, but not invariant by Proposition $\mathrm{B}$, and $C_{b} \cap C=\varnothing$ by Proposition 1 , we have $C_{b} \cap \partial$ (int $J_{b}$-int $C$ ) $=\varnothing$. Thus $C_{b}$ is an internal limit cycle of $\pi_{b}$ contained in int $J_{b} \subset U$. For c sufficiently large $y_{1} \pi_{b}[c, \infty) \subset \operatorname{int} J_{b}$ and therefore $y_{1} \pi_{b}[c, \infty) \subset \operatorname{int} J_{b}-\overline{\operatorname{int} C_{b}}$. The Poincaré-Bendixson Theorem now implies the existence of an external limit cycle. This completes the proof.

In a similar manner it can be shown that

Proposition 8. Let $C$ be an external negative limit cycle of $\pi_{a}$. Then, given any outer neighborhood $U$ of $C$, there exists an $\varepsilon>0$ such that, for each $b \in[a-\varepsilon, \alpha]$, $U$ contains both an external negative limit cycle and an internal negative limit cycle of $\pi_{b}$ (the two cycles may coincide). A similar result holds for $C$ an internal negative limit cycle and $b \in[a, a+\varepsilon]$.

Lemma 9. Let $D_{1}$ and $D_{2}$ be cycles of a complete family such that $D_{1} \subset \operatorname{int} D_{2}$ and that int $D_{2}$-int $D_{1}$ contains no critical points. 
If $C_{1}$ and $C_{2}$ are distinct cycles in int $D_{2}-\operatorname{int} D_{1}$, then $C_{1} \subset \operatorname{int} C_{2}$ or $C_{2} \subset \operatorname{int} C_{1}$.

Proof. Since int $D_{2}-\operatorname{int} D_{1}$ contains no critical points, we must have $D_{1} \subset \operatorname{int} C_{i}, i=1,2$. Thus $\operatorname{int} C_{1} \cap \operatorname{int} C_{2} \neq \varnothing$. Then $\operatorname{int} C_{1} \subset \operatorname{int} C_{2}$ or int $C_{1} \cap$ ext $C_{2} \neq \varnothing$. In the first case $\overline{\operatorname{int} C_{1}} \subset \overline{\operatorname{int} C_{2}}$. Therefore $C_{1} \subset \operatorname{int} C_{2}$ or $C_{1} \cap C_{2} \neq \varnothing$. The latter is impossible by Proposition 1 . In the second case, $\partial$ (int $C_{2}$ ) $\cap$ int $C_{1} \neq \varnothing$. Therefore $C_{2} \cap \operatorname{int} C_{1} \neq \varnothing$ and $C_{2} \subset \operatorname{int} C_{1}$ since $\operatorname{int} C_{1}$ is either positively invariant or negatively invariant for the system containing $C_{2}$ (Proposition 3).

Let $D_{1}$ and $D_{2}$ be as in the statement of Lemma 9. Then

Lemma 10. If $C_{1}$ and $C_{2}$ are distinct cycles in int $D_{2}$-int $D_{1}$ such that $C_{1} \subset \operatorname{ext} C_{2}$, then $C_{2} \subset \operatorname{int} C_{1}$

Proof. By Lemma 9, $C_{2} \subset \operatorname{int} C_{1}$ or $C_{1} \subset \operatorname{int} C_{2} . C_{1}$ cannot be contained in both $\operatorname{int} C_{2}$ and ext $C_{2}$. Therefore $C_{2} \subset \operatorname{int} C_{1}$.

In a topological space $X$, it is possible to define limits of nets of subsets $X_{i} \subset X$ as follows. Let $\lim \inf X_{i}$ consist of all limits of nets of points $x_{i} \in X_{i}$; let $\lim \sup X_{i}$ consist of all limits of subnets of points $x_{i} \in X_{i}$. Obviously $\lim \inf X_{i} \subset \lim \sup X_{i}$. If equality holds, the net $X_{i}$ is said to converge to its limit and we write

$$
\lim X_{i}=\lim \inf X_{i}=\lim \sup X_{i} .
$$

DEFINITION 11. A net $\left(R^{2}, \pi_{i}\right), i$ contained in a directed set containing 0 , of dynamical system is called regular if

(i ) $\pi_{i} \rightarrow \pi_{0}$ in the sense that if $x_{i} \rightarrow x$ and $t_{i} \rightarrow t$ then $x_{i} \pi_{i} t_{i} \rightarrow x \pi_{0} t$.

(ii) critical points are independent of the index $i$.

(iii) to each noncritical point $x$ there corresponds a subset $T$ of $R^{2}$ which is a transversal with respect to each $\pi_{i}$ and contains $x$ as a nonend point.

In [3] the following theorem is proved.

THEOREM D. Let $\left(R^{2}, \pi_{i}\right)$ be a regular net of dynamical systems. Let $C_{i}\left(x_{i}\right)$ be a cycle of $\left(R^{2}, \pi_{i}\right)$ with fundamental period $\tau_{i}\left(x_{i}\right)$. If $\lim \inf C_{i}\left(x_{i}\right) \neq \varnothing$, then

(1) If $\tau_{i}\left(x_{i}\right) \rightarrow 0$, then $\lim C_{i}\left(x_{i}\right)$ exists and is a single critical point.

(2) If $\lim \inf C_{i}\left(x_{i}\right)$ intersects a cycle $C_{0}(x)$, then $\tau_{i}\left(x_{i}\right) \rightarrow \tau_{0}(x)$ and $\lim C_{i}\left(x_{i}\right)=C_{0}(x)$. 
(3) If $\lim \inf C_{i}\left(x_{i}\right)$ intersects a noncyclic trajectory, then $\tau_{i}\left(x_{i}\right)$ $\rightarrow+\infty$.

Definition 12. Let $C_{a}(x)$ be a cycle of $\pi_{a}$. Then $\tau_{a}(x)$ will denote the fundamental period of $x$ with respect to $\pi_{a}$.

Proposition 13. Let $C$ be an external limit cycle of $\pi_{a}$. There exists an outer neighborhood $U$ of $C$ and an $\varepsilon>0$ such that $U$ consists entirely of periodic points of the systems $\pi_{b}, b \in[a, a+\varepsilon] . \quad A$ similan result holds for $C$ an internal limit cycle and $b \in[a-\varepsilon, a]$.

Proof. Let $x \in C$ and $V$ be an outer neighborhood of $C$ which contains no other cycles of $\pi_{a}$ or critical points and such that $V \cup$ int $C$ is simply connected. Moreover, by Theorem $\mathrm{D}, V$ may be chosen along with a $\sigma>0$ such that if $C_{b}(y)$ is a cycle of $\pi_{b}$ in $V$ with $|b-a|<\sigma$, then $\left|\tau_{a}(x)-\tau_{b}(y)\right|<1 / 2 \tau_{a}(x)$. By Proposition 7 there is an $\varepsilon, 0<\varepsilon<\sigma$ such that, for each $b \in[a, a+\varepsilon], V$ contains a cycle of $\pi_{b}$. Thus the fundamental periods cycles of $\pi_{a+\varepsilon}$ which lie in $V$ are contained in $\left[1 / 2 \tau_{a}(x), 3 / 2 \tau_{a}(x)\right]$. This, Theorem $\mathrm{D}$ with each $i=a+\varepsilon$, and the fact that cycles of distinct systems do not intersect imply that there is a cycle $D$ of $\pi_{a+\varepsilon}$ in $V$ such that int $D$-int $C$ contains no cycle of $\pi_{a+\varepsilon}$. Set $U=\overline{\operatorname{int} D}-\operatorname{int} C$. $U$ is an outer neighborhood of $C$ by Lemma 10 . Let $A$ denote the set of periodic points of $\pi_{b}, b \in[a, a+\varepsilon]$, which are contained in $U$. We will show that $A=U$. Assume the contrary that there exists a $w \in U-A$ and consider the sets

$$
\begin{aligned}
& F=\left\{\overline{\operatorname{int} C_{b}(y)}: \quad y \in A, C_{b}(y) \text { a cycle }, w \in \operatorname{ext} C_{b}(y)\right\} \\
& G=\cup F .
\end{aligned}
$$

Since $w \in U$, we have $w \in \operatorname{ext} C=\operatorname{ext} C_{a}(x)$, so that $F \neq \varnothing$. If $C_{b}(y) \subset G \subset U$, then $\tau_{b}(y) \in\left[1 / 2 \tau_{a}(x), 3 / 2 \tau_{a}(x)\right]$. Proposition 7 and Theorem $\mathrm{D}$ now imply, respectively, that $\partial G \cap \operatorname{ext} C \neq \varnothing$ and $\partial G$ consists entirely of periodic points. Lemma 9 implies that $\partial G \cap \operatorname{ext} C$ is a cycle $C_{d}(z)$ where $z \in U$ and $d \in[a, a+\varepsilon]$. Moreover, since $w \in \operatorname{ext} C_{b}(y)$ for each $\overline{\operatorname{int} C_{b}(y)}$ in $F$ and $C_{b}(w)$ is not a cycle for any $b \in[a, a+\varepsilon]$, we have $w \in \operatorname{ext} C_{d}(z) . d \neq a$ since $C_{d}(z)=\partial G \cap \operatorname{ext} C \subset V$ and the only cycle of $\pi_{a}$ in $V$ is $C$. Since $U \neq A, C_{d}(z) \neq D$. Hence $d \neq a+\varepsilon$. Also, by the construction of $C_{d}(z)$, there is no cycle $B$ of $\pi_{b}, b \in[a, a+\varepsilon]$, in $U$ such that $C_{d}(z) \subset \operatorname{int} B$ and $w \in \operatorname{ext} B$. Thus $C_{d}$ is either an external limit cycle or an external negative limit cycle, [2, VIII, 3. 3]. Proposition 7 or 8 , respectively, now implies the existence of a $c \in[a, a+\varepsilon]$ such that a cycle $C_{1}$ of $\pi_{c}$ has the property that $C_{d}(z) \subset \operatorname{int} C_{1}$ and $w \in \operatorname{ext} C_{1}$. This contradiction implies $A=U$. This completes the proof. 
In a similar manner it can be shown that

Proposition 14. Let $C$ be an external negative limit cycle of $\pi_{a}$. There exists an outer neighborhood $U$ of $C$ and an $\varepsilon>0$ such that $U$ consists entirely of periodic points of the systems $\pi_{b}, b \in[a-\varepsilon, a]$. $A$ similar result holds for $C$ an internal negative limit cycle and $b \in[a, a+\varepsilon]$.

\section{REFERENCES}

1. G. D. F. Duff, Limit-cycles and rotated vector fields, Ann. of Math. 57 (1953), 1531.

2. Otomar Hajek, Dynamical Systemsion the Plane, Academic Press, London, 1968.

3. Roger C. McCann, Cycle periods of perturbed dynamical systems, (to appear)

4. George Seifert, Rotated vector fields and an equation for ralaxation oscillations, Contributions to the Theory of Nonlinear Oscillations 4, Princeton, 1958, 125-139.

Received December 22, 1969.

Case Western Reserve University

Cleveland, OHio 


\section{PACIFIC JOURNAL OF MATHEMATICS}

\section{EDITORS}

H. SAmelson

Stanford University

Stanford, California 94305

RICHARd PieRCe

University of Washington

Seattle, Washington 98105

\author{
J. DugundJI \\ Department of Mathematics \\ University of Southern California \\ Los Angeles, California 90007 \\ RICHARD ARENS \\ University of California \\ Los Angeles, California 90024
}

ASSOCIATE EDITORS
E. F. BECKENBACH
B. H. NeumanN
F. WOLF
K. YosHIDA

\section{SUPPORTING INSTITUTIONS}

UNIVERSITY OF BRITISH COLUMBIA

CALIFORNIA INSTITUTE OF TECHNOLOGY

UNIVERSITY OF CALIFORNIA

MONTANA STATE UNIVERSITY

UNIVERSITY OF NEVADA

NEW MEXICO STATE UNIVERSITY

OREGON STATE UNIVERSITY

UNIVERSITY OF OREGON

OSAKA UNIVERSITY

UNIVERSITY OF SOUTHERN CALIFORNIA
STANFORD UNIVERSITY

UNIVERSITY OF TOKYO

UNIVERSITY OF UTAH

WASHINGTON STATE UNIVERSITY

UNIVERSITY OF WASHINGTON

AMERICAN MATHEMATICAL SOCIETY CHEVRON RESEARCH CORPORATION TRW SYSTEMS

NAVAL WEAPONS CENTER 


\section{Pacific Journal of Mathematics}

\section{Vol. 35, No. $1 \quad$ September, 1970}

B. D. Arendt and C. J. Stuth, On the structure of commutative periodic semigroups ..................................... 1

B. D. Arendt and C. J. Stuth, On partial homomorphisms of semigroups .... 7

Leonard Asimow, Extensions of continuous affine functions ............ 11

Claude Elias Billigheimer, Regular boundary problems for a five-term recurrence relation ................................... 23

Edwin Ogilvie Buchman and F. A. Valentine, A characterization of the

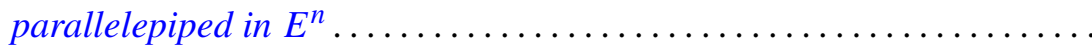
53

Victor P. Camillo, A note on commutative injective rings ............ 59

Larry Jean Cummings, Decomposable symmetric tensors ............. 65

J. E. H. Elliott, On matrices with a restricted number of diagonal values ... 79

Garth Ian Gaudry, Bad behavior and inclusion results for multipliers of type

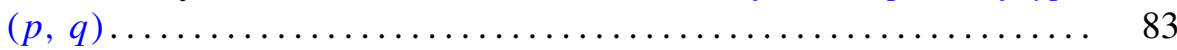

Frances F. Gulick, Derivations and actions .................. 95

Langdon Frank Harris, On subgroups of prime power index . . . . . . . . 117

Jutta Hausen, The hypo residuum of the automorphism group of an abelian

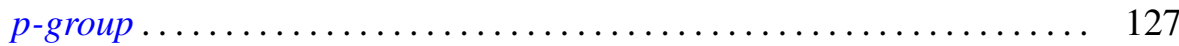

R. Hrycay, Noncontinuous multifuctions . ................... 141

A. Jeanne LaDuke, On a certain generalization of $p$ spaces ............ 155

Marion-Josephine Lim, Rank preservers of skew-symmetric matrices...... 169

John Hathway Lindsey, II, On a six dimensional projective representation of the Hall-Janko group ................................... 175

Roger McCann, Transversally perturbed planar dynamical systems . . . . . 187

Theodore Windle Palmer, Real $C^{*}$-algebras ................... 195

Don David Porter, Symplectic bordism, Stiefel-Whitney numbers, and a

Novikov resolution ....................................... 205

Tilak Raj Prabhakar, On a set of polynomials suggested by Laguerre

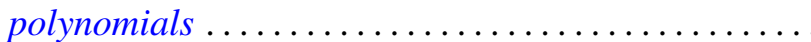

B. L. S. Prakasa Rao, Infinitely divisible characteristic functionals on locally convex topological vector spaces...................

John Robert Reay, Caratheodory theorems in convex product structures ...

Allan M. Sinclair, Eigenvalues in the boundary of the numerical range. .

David R. Stone, Torsion-free and divisible modules over matrix rings ....

William Jennings Wickless, A characterization of the nil radical of a

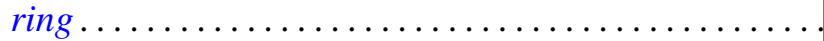

